\title{
Fatores associados ao bem-estar subjetivo de crianças e adolescentes em situação de rua
}

\author{
Rebeca Fernandes Ferreira Lima \\ Normanda Araujo de Morais \\ Universidade de Fortaleza \\ Fortaleza, CE, Brasil
}

\begin{abstract}
Resumo
Buscou-se caracterizar o bem-estar subjetivo de crianças e adolescentes em situação de rua de três capitais brasileiras: Fortaleza, Porto Alegre e Salvador, verificando os fatores a ele associados (idade, sexo, eventos estressores e rede de apoio). Participaram 111 jovens $(M=14,18$ anos; $D P=2,4)$, sendo a maioria $(\mathrm{n}=90 ; 81,1 \%)$ meninos. Utilizou-se o Inventário de Eventos Estressores, Mapa dos Cinco Campos, Escala de Satisfação de Vida e Escalas de Afeto Positivo e Negativo. Os participantes avaliaram positivamente a satisfação de vida e relataram mais afetos positivos que negativos, embora tenham vivenciado eventos estressores. Satisfação de vida associou-se negativamente com idade e afetos negativos associaram-se positivamente ao impacto dos eventos estressores e negativamente ao fator de proximidade da rede de apoio. Discute-se que as adversidades não afetaram a expressão de afetos positivos e satisfação de vida, bem como a importância da rede de apoio para promoção de bem-estar.
\end{abstract}

Palavras-chave: Bem-estar subjetivo; Eventos estressores; Rede de apoio; Situação de rua.

\section{Factors associated with the subjective well-being of children and adolescents in street situation}

\begin{abstract}
This study aimed to characterize the subjective well-being of children and adolescents in street situation from: Fortaleza, Porto Alegre and Salvador, verifying the factors associated with it (age, gender, stressful events and support network). Participants were 111 young people $(M=14.18$ years, $S D=2.4)$, the majority $(n=90 ; 81.1 \%)$ were male. Stressful Events Inventory, the Five Field Map, Life Satisfaction Scale and Positive and Negative Affect Scale were used. Participants positively evaluated the life satisfaction and reported more positive emotions than negative, although they have often experienced the stressful events. There was a negative correlation between age and life satisfaction and negative affect was positively associated to the impact of stressful events and negatively to the proximity factor of the support network. We discuss that adversities do not affect the expression of positive affect and life satisfaction, and the importance of the support network for well-being promotion.
\end{abstract}

Keywords: Subjective well-being; Stressful events; Support network; Street situation.

\section{Factores asociados con el bienestar subjetivo de los niños y adolescentes en situación de la calle}

Resumen

Este estudio tuvo como objetivo caracterizar el bienestar subjetivo de los niños y adolescentes que viven en la calle de tres ciudades de Brasil: Fortaleza, Porto Alegre y Salvador, la verificación de los factores asociados a el bienestar subjetivo (edad, sexo, eventos estresantes; red de apoyo). Participaron 111 jóvenes ( $M=14,18$ años, $\mathrm{SD}=2.4)$, la mayoría ( $\mathrm{n}=90 ; 81,1 \%)$ eran de sexo masculino. Se utilizó el Inventario de Eventos Estresantes, Mapa de Cinco Campos, Escala de Satisfacción con la Vida y Escala de Afecto Positivo y Negativo. Los participantes evaluaron positivamente la satisfacción con la vida y reportaron más emociones positivas que negativas, aunque a menudo han experimentado los eventos estresantes. Se observaron una correlación negativa entre la edad y la satisfacción con la vida y afecto negativo se asoció positivamente con el impacto de los eventos estresantes y negativamente con el factor de la proximidad de la red de apoyo. Se discute que las adversidades no afectan a la expresión de afecto positivo y satisfacción con la vida y la importancia de la red de apoyo para la promoción de bienestar.

Palabras clave: Bienestar subjetivo; Eventos estresantes; Red de apoyo; Situación de calle. 
A investigação sobre o bem-estar subjetivo (BES) é uma área emergente na psicologia do desenvolvimento. Caracteriza-se como um avanço teórico, metodológico e ético para a proposição de intervenções adequadas à busca da qualidade de vida dos jovens em diferentes contextos de desenvolvimento. O BES é definido como uma categoria de fenômenos que inclui respostas emocionais, satisfações de domínios específicos da vida e julgamentos globais da satisfação de vida. Portanto, refere-se à avaliação individual das experiências de vida, tanto de forma emocional quanto cognitiva (Rees, Goswami, \& Bradshaw, 2010).

A dimensão emocional, composta pelos afetos positivos e negativos, compreende as respostas circunstanciais do indivíduo aos eventos de vida. Os afetos positivos representam as emoções agradáveis, tais como alegria e prazer. Já os afetos negativos indicam as emoções desagradáveis, como tristeza e raiva. A dimensão cognitiva refere-se ao julgamento individual da satisfação de vida com base em um padrão social ou comparação de circunstâncias passadas da vida. Assim, o BES é um construto que é determinado pela percepção subjetiva do indivíduo e caracteriza-se por ser multidimensional, isto é, refere-se ao julgamento global da satisfação de vida e dimensões específicas, por exemplo, a família, escola e vizinhança (Segabinazi, Giacomoni, Dias, Teixeira, \& Moraes, 2010).

Pesquisas com jovens em situação de rua, tradicionalmente, os descreveram destacando-se as vulnerabilidades em situações envolvendo alto risco para vários resultados desenvolvimentais negativos (Morais, Raffaelli, \& Koller, 2012). Entretanto, entende-se que o estudo de indicadores positivos nessa população pode contribuir para uma perspectiva mais positiva sobre os mesmos e para melhorias em sua qualidade de vida. Por exemplo, pesquisas com jovens em vulnerabilidade indicam que relações afetivas significativas atuam como proteção, resultando em melhores níveis de ajustamento psicossocial, satisfação e bem-estar (Morais, Koller, \& Raffaelli, 2012; Siqueira \& Dell'Aglio, 2010; Schütz, Sarriera, Bedin, \& Montserrat, 2014).

No que se refere ao BES, nota-se uma escassez de estudos com adolescentes quando comparados aos realizados com adultos (Casas et al., 2013). Muitas pesquisas com jovens foram desenvolvidas em contexto anglo-saxão, tendo como interesse estudar população clínica e estudantes. Trata-se de uma alarmante evidência, posto que o BES é influenciado por indicadores próprios de cada cultura, variando, portanto, entre as sociedades (Diener, 2012).
Pesquisas que consideram a importância de se investigar diferentes contextos socioculturais e população em vulnerabilidade, em geral, localizam-se na América Latina. Nesta seara encontram-se estudos que utilizam diferentes variáveis para investigação do BES, relacionadas à felicidade, qualidade de vida, satisfação de vida e saúde mental. Quanto às diferenças socioculturais, verificam-se resultados de maiores níveis do BES em comparação a valores normativos dos países ocidentais; diminuição progressiva do bem-estar com a idade; a influência da cultura de cada país no nível de BES; e que não há consenso quanto à relação entre satisfação de vida e sexo (Casas et al., 2013; Sarriera et al., 2012). Quanto à população em vulnerabilidade, Cervantes e Sosa (2015) investigaram o BES e a saúde mental de jovens em situação de rua. Indicou-se que as participantes tiveram sintomas de ansiedade e depressão que estiveram associados a maior frequência de afetos negativos.

No Brasil, alguns estudos sobre o BES de jovens em vulnerabilidade foram encontrados, porém se verificou que, em geral, esses estudos foram realizados em escolas, instituições de acolhimento e famílias (Morais et al., 2012; Poletto \& Koller, 2011; Siqueira et al., 2010; Schütz et al., 2014). Resultados de comparação entre esses contextos, em sua maioria, apontaram que os jovens em vulnerabilidade (em acolhimento institucional) apresentam altos escores de BES. Nota-se que a experiência de eventos adversos desencadeia um alto nível de afetos negativos, mas essas circunstâncias não resultam necessariamente em diminuição dos níveis de afetos positivos e satisfação de vida. No entanto, apenas uma pesquisa foi realizada com jovens em situação de rua. Esta se deteve a uma amostra de jovens gaúchos e não analisou o domínio da satisfação de vida (Morais et al., 2012; Morais et al., 2012).

Enfatiza-se a realização de pesquisas sobre o BES em diferentes contextos, posto que variáveis presentes nas diferentes culturas e subculturas interferem na avaliação do BES. Acrescenta-se, ainda, a importância da investigação do BES relacionado a outras variáveis, tais como eventos estressores e rede de apoio, visto que estas variações podem enriquecer a investigação sobre o BES e suas implicações. Os eventos estressores referem-se a situações associadas à alta probabilidade de resultados desenvolvimentais negativos (Masten \& Garmezy, 1985) e a rede de apoio consiste em um conjunto de sistemas e de pessoas significativas que compõem os elos de relacionamento recebidos e percebidos do indivíduo (Brito \& Koller, 1999).

Com base nas considerações acima, este estudo objetivou caracterizar o bem-estar subjetivo de crianças e adolescentes em situação de rua de três 
capitais brasileiras: Fortaleza, Porto Alegre e Salvador, verificando os fatores a ele associados (idade, sexo, eventos estressores e rede de apoio). A hipótese a ser testada é de que o BES tem correlação significativa positiva com rede de apoio e negativa com idade e eventos estressores.

\section{Método}

\section{Delineamento e Participantes}

Trata-se de um estudo transversal e exploratório (com caráter analítico) realizado com 111 crianças e adolescentes de ambos os sexos com experiência de vida na rua, isto é, aqueles que utilizavam a rua para trabalho, lazer e/ou moradia ou que haviam estado neste espaço há pelo menos dois anos da coleta de dados. As idades variaram entre 09 e 18 anos $(M=14,18$ anos; $D P=2,4)$, sendo $90(81,1 \%)$ meninos e $21(18,9 \%)$ meninas. A maioria $(\mathrm{n}=89 ; 80,18 \%)$ estava em acolhimentos institucionais; seguido pelos serviços abertos, instituições que funcionam de forma provisória, sendo permitido ao adolescente frequentar a rua $(\mathrm{n}=18 ; 16,22 \%)$; e um menor número foi encontrado diretamente na rua $(n=4 ; 3,6 \%)$. Do total, 45 foram entrevistados em Fortaleza (40,5\%), 40 em Salvador (36\%) e 26 em Porto Alegre $(23,4 \%)$. As cidades foram escolhidas por conveniência, por se tratarem das cidades de origem dos coordenadores da pesquisa.

\section{Instrumentos}

Inventário de Eventos Estressores (Raffaelli, Koller, \& Morais, 2007) composto por 22 itens, como "morte do pai ou da mãe" e "parou de estudar". Esse inventário foi adaptado de Kristensen, Leon, D'Incao e Dell'Aglio (2004) para o uso com jovens em situação de rua, o qual mostrou adequada consistência interna de 0,77 para o alpha de Cronbach, neste estudo. Os participantes indicaram a ocorrência do evento como sim/não e avaliaram quanto ao impacto experienciado (5 pontos; 1 - nada estressante a 5 - totalmente estressante). A ocorrência foi calculada a partir da soma do número de respostas "sim" aos itens do inventário. A média do impacto total do inventário foi calculada através da divisão da soma total dos valores de impacto atribuídos para cada evento já ocorrido pelo número total de eventos com impacto válido.

Mapa dos Cinco Campos (Samuelsson, Thernlund, \& Ringström, 1996, adaptado por Hoppe, 1998) é comporto por cinco círculos concêntricos divididos em partes iguais, que correspondem a dimensões da rede de apoio dos jovens ("família", "escola", "instituições", "rua" e "amigos"). O participante é convidado a acrescentar no mapa as pessoas que fazem parte da sua rede de apoio, desde as mais próximas afetivamente àquelas com as quais o jovem tem relacionamento insatisfatório. O mapa avalia o tamanho da rede de apoio (número de contatos) e fator de proximidade, o qual representa a qualidade dos vínculos estabelecidos ( 1 - muito próximo a 5 - totalmente distante). Para o cálculo do fator de proximidade, o número de pessoas no primeiro nível foi multiplicado por oito; no segundo nível por quatro; no terceiro nível por dois; no quarto nível por um e no quinto nível por zero. Em seguida, o somatório desse cálculo foi dividido pelo número total de pessoas citadas no mapa.

Escala de Satisfação de Vida (Diener, Emmons, Larsen, \& Griffin 1985; Koller \& Hutz, 1996). Composta por 5 itens, como "a sua vida está próxima do seu ideal?" e "você está satisfeito com sua vida?". As respostas pontuaram em uma escala de 5 pontos ( 1 - discordo fortemente a 5 - concordo fortemente). Neste estudo, a escala obteve uma adequada consistência interna (alpha de Cronbach $=0,72$ ).

Escalas de Afeto Positivo e Negativo (Giacomoni, 2002). Composta por 34 itens com estrutura bifatorial de afeto positivo (17 itens; 1 - nem um pouco a 5 - muitíssimo) e afeto negativo (17 itens; 1 - nem um pouco a 5 - muitíssimo). Os afetos positivos são: divertido, contente, carinhoso, feliz, animado, alegre, participativo, satisfeito, esperto, forte, amoroso, corajoso, decidido, esforçado, cuidadoso, delicado e interessado. Já os afetos negativos são: irritado, magoado, perturbado, nervoso, triste, furioso, culpado, preocupado, chateado, humilhado, deprimido, desanimado, incomodado, assustado, envergonhado, impaciente e amedrontado. Neste estudo, as escalas obtiveram adequada consistência interna (Escala de Afeto Positivo: alpha de Cronbach = 0,86; Escala de Afeto Afeto Negativo: alpha de Cronbach $=0,86$ ).

\section{Procedimentos de Coleta de Dados}

A coleta de dados dessa pesquisa teve como base a metodologia da Inserção Ecológica, a qual está baseada na Abordagem Bioecológica do Desenvolvimento Humano e visa a inserção do pesquisador no ambiente natural de pesquisa, a fim de favorecer a proximidade com os jovens e, assim, responder às questões de pesquisa (Cecconello \& Koller, 2003; Prati et al., 2008).

Inicialmente, identificaram-se locais potenciais de recrutamento, ou seja, onde havia prestação de serviços socioassistenciais para jovens em situação de rua. Posteriormente, as instituições de acolhimento, serviços abertos e espaços da rua que foram identificados como locais de encontro regular 
dos jovens foram selecionados para o recrutamento de participantes, pois seria possível estabelecer vínculos entre pesquisadores e jovens ao passo que estes frequentavam com assiduidade esses ambientes. Por fim, a equipe de pesquisa se inseriu nesses espaços, tendo início a inserção ecológica a qual se estendeu por seis meses. Neste período, a equipe frequentou de duas a três vezes por semana os locais selecionados, participando de conversas e atividades informais com os jovens, profissionais (principalmente, educadores sociais que os acompanhavam rotineiramente) e pessoas do entorno que faziam parte da rede social dos jovens, como taxistas, trabalhadores do comércio e adultos em situação de rua. As impressões e sentimentos dos pesquisadores, descrição de ambientes, situações e trechos de fala foram registradas em diários de campo, que auxiliaram na interpretação dos resultados. Após identificação e abordagem dos participantes, bem como da anuência dos mesmos em participarem, os instrumentos foram aplicados individualmente com duração média de 35 minutos.

\section{Procedimentos de Análise de Dados}

Utilizou-se o Statistical Package for Social Sciences - SPSS (versão 19) para análise de dados. Adotou-se um $p<0,05$ como nível de significância crítico para todas as análises. Os resultados foram organizados em tópicos de acordo com os diferentes objetivos de análise dos dados. Assim, expôs-se a descrição do BES, eventos estressores e rede de apoio, seguido das análises de comparação, que dependendo do grau de normalidade das variáveis, utilizou-se o Teste $t$ de Student e Mann-Whitney, para até duas amostras e ANOVA e Kruskal-Wallis, para mais de duas amostras. Após, realizaram-se as análises de correlação e regressão linear entre as variáveis do estudo.

\section{Aspectos Éticos}

Este estudo ocorreu mediante autorização do Comitê de Ética da Universidade de Fortaleza (Protocolo 2011023). Obteve assinatura do Termo de Concordância do Ministério Público e das instituições em que ocorreu a inserção dos pesquisadores. Para cada jovem recrutado foi explicitado os objetivos de pesquisa, a natureza voluntária do estudo, garantia de sigilo, confidencialidade dos dados, a liberdade de desistência ao longo da pesquisa e demais procedimentos éticos pautados na Resolução 196/1996 do Conselho Nacional de Saúde (vigente à época de aprovação da pesquisa).

\section{Resultados}

\section{Descrições do Bem-Estar Subjetivo: Satisfação de Vida, Afetos Positivos e Afetos Negativos}

Quanto à satisfação de vida, conforme mostra a Tabela 1, verificou-se que os participantes tenderam a apresentar médias que podem ser avaliadas como altas, visto que a escala possui variação de 5 pontos. Esse resultado indicou a tendência dos jovens em situação de rua avaliar positivamente a satisfação de vida. A comparação entre as cidades mostrou que as médias encontradas em Fortaleza foram nominalmente maiores, embora não tenha sido encontrada diferença significativa $\left[\chi^{2}(2, N=108)=3,53, p=0,17\right]$.

TABELA 1

Médias e Desvios-Padrão das Variáveis Idade, Eventos Estressores (Número e Impacto), Rede de Apoio (Contatos e Fator de Proximidade), Satisfação de Vida, Afeto Positivo e Afeto Negativo na Avaliação dos Participantes de Fortaleza, Porto Alegre e Salvador e no Total das Três Cidades

\begin{tabular}{|c|c|c|c|c|}
\hline \multirow{3}{*}{ Variáveis } & \multicolumn{3}{|c|}{ Cidades } & \multirow{2}{*}{ Total } \\
\hline & Fortaleza & Porto Alegre & Salvador & \\
\hline & $M(S D)$ & $M(S D)$ & $M(S D)$ & $M(S D)$ \\
\hline Idade & $13,76(2,71)$ & $15,15(1,89)$ & $14,03(2,19)$ & $14,18(2,4)$ \\
\hline Satisfação de Vida & $3,41(0,92)$ & $2,84(1,22)$ & $3,26(1,02)$ & $3,22(1,05)$ \\
\hline Afeto Positivo & $3,68(0,71)$ & $3,34(0,76)$ & $3,32(0,9)$ & $3,47(0,81)$ \\
\hline Afeto Negativo & $2,75(0,79)$ & $2,83(0,91)$ & $2,89(1,05)$ & $2,82(0,91)$ \\
\hline Número & $10,50(4,59)$ & $9,24(3,72)$ & $7,52(3,32)$ & $9,09(4,13)^{* *}$ \\
\hline Impacto & $2,94(0,68)$ & $2,95(1)$ & $2,88(0,86)$ & $2,92(0,83)$ \\
\hline Contatos & $27,34(10,03)$ & $21,08(9,14)$ & $17,68(18,94)$ & $22,65(14,66)^{* * *}$ \\
\hline Fator de Proximidade & $5,13(1,7)$ & $5,58(1,51)$ & $5,51(1,85)$ & $5,34(1,73)$ \\
\hline
\end{tabular}

Nota: Resultado significativo para diferença entre cidades.

$* p<0,05, * * p<0,01, * * * p<0,001$. 
Quanto aos afetos positivos (ver Tabela 1), verificam-se médias que também podem ser avaliadas como altas, visto que a escala varia em 5 pontos. Isto demostra que os participantes tenderam a indicar uma alta frequência de afetos positivos no seu cotidiano. Os afetos positivos mais frequentes foram: interessado, contente e esperto. Já os menos frequentes foram: satisfeito, delicado e carinhoso. Por cidade, verificou-se uma tendência das médias mais altas serem encontradas em Fortaleza, entretanto não se observou diferença significativa $(F=2,38, p=0,09)$.

Observa-se que as médias atribuídas aos afetos negativos, por cidade e no total das três capitais, sugerem que os participantes tendem a sentir com menor frequência os afetos negativos em relação aos afetos positivos (ver Tabela 1). Os afetos negativos mais frequentes foram: preocupado, magoado e nervoso. Já os menos frequentes foram: amedrontado, deprimido e humilhado. Observou-se uma tendência das médias mais altas serem encontradas em Salvador, embora não se tenha encontrado diferença significativa $(F=2, p=0,81)$.

\section{Caracterização dos Eventos Estressores (Número e Impacto)}

Quanto ao número de eventos estressores (ver Tabela 1), os participantes apresentaram uma média geral de 9,09 eventos $(D P=4,13)$, cuja amplitude variou de zero a dezenove eventos. Os eventos mais frequentes foram "começar a dormir em um lugar novo", "viver/morar com novas pessoas (familiares/ amigos), "parar de ver um amigo próximo ou grupo de amigos" e "começar a frequentar uma instituição". No que se refere à avaliação do impacto, verificou-se média de "um pouco estressante" a "mais ou menos estressante" $(M=2,92 ; D P=0,83)$. As maiores médias de impacto foram atribuídas a "foi vítima de violência sexual", "morte do pai ou da mãe", "morte de um amigo próximo ou parceiro romântico" e "foi vítima de violência física". Na comparação entre cidades, observou-se diferença significativa para frequência dos eventos $(F=5,81, p<0,01)$. Enfatiza-se que Fortaleza apresentou maior ocorrência de eventos quando comparada a Salvador. Não se observou diferença significativa para impacto dos eventos estressores $(F=0,57, p=0,94)$.

\section{Caracterização da Rede de Apoio (Contatos e Fator de Proximidade)}

Conforme apresenta a Tabela 1, os participantes das três cidades tiveram média geral de 22,65 $(D P=14,66)$ contatos na rede de apoio. $\mathrm{Na}$ comparação entre cidades, verificou-se que médias mais altas foram encontradas em Fortaleza, com diferença significativa $\left[\chi^{2}(2, N=82)=19,77, p<0,001\right]$. Enfatiza-se que Fortaleza teve mais contatos na rede de apoio quando comparada a Salvador $(U=238,5, z=-4,36, p<0,001)$. Quanto ao fator de proximidade, os participantes, no total das três cidades, apresentaram uma rede de apoio que pode ser avaliada como de "média força", de acordo com Siqueira, Tubino, Schwarz e Dell' Aglio (2009). Quando se comparou as médias gerais do fator de proximidade do mapa entre as cidades, verificou-se que as médias de Porto Alegre foram nominalmente maiores, embora não se observou diferença significativa $(F=0,54, p=0,59)$.

\section{Comparações dos Escores de Bem-Estar Subjetivo, Eventos Estressores e Rede de Apoio no que se Refere às Variáveis Sociodemográficas Idade e Sexo}

Quando se comparou satisfação de vida por idade, verificou-se que os participantes com menor idade tenderam a apresentar médias mais altas de satisfação de vida $(F=5,39, p<0,01)$. Os afetos positivos, afetos negativos, eventos estressores e rede de apoio não apresentaram níveis de significância quando comparados à idade. No que se refere ao sexo, verificou-se que os meninos tenderam a apresentar médias mais altas de satisfação de vida quando comparados às meninas $(U=601,5, z=-1,97, p=0,04)$. Em relação à média do impacto dos eventos estressores, verificou-se que as meninas apresentaram médias mais altas de impacto quando comparadas aos meninos $(t=2,67 ; d f=93 ; p<0,01)$. As demais variáveis deste estudo quando comparadas entre os sexos não apresentaram diferença significativa.

\section{Correlações do Bem-estar Subjetivo às Variáveis Eventos Estressores e Rede de Apoio}

Conforme apresenta a Tabela 2, verificou-se que afeto positivo e eventos estressores correlacionaramse positivamente com número de contatos da rede de apoio, isto é, quanto maior o número de contatos, médias mais altas de afeto positivo e número de eventos estressores foram observadas. $\mathrm{O}$ afeto negativo teve correlação positiva com número e impacto dos eventos estressores, indicando que quanto maior a ocorrência de eventos estressores e suas médias de impacto, maiores são as médias de afeto negativo. Nas correlações entre os componentes do BES verificou-se que satisfação de vida se correlacionou positivamente com afeto positivo e negativamente com afeto negativo. Isto indicou que quanto mais altas as médias de satisfação de vida, médias mais altas são apresentadas nos afetos positivos, 
TABELA 2

Correlações entre Satisfação de Vida, Afeto Positivo e Afeto Negativo com Eventos Estressores (Número e Impacto) e Rede de Apoio (Contatos e Fator de Proximidade)

\begin{tabular}{|c|c|c|c|c|c|c|c|}
\hline Variáveis & 1 & 2 & 3 & 4 & 5 & 6 & 7 \\
\hline 1. Satisfação de Vida ${ }^{\mathrm{b}}$ & - & $0,3 * *$ & $-0,2 *$ & $-0,94$ & $-0,08$ & 0,2 & 0,13 \\
\hline 2. Afeto Positivo ${ }^{a}$ & $0,3 * *$ & - & 0,11 & 0,09 & 0,13 & $0,29 * *$ & $-0,05$ \\
\hline 3. Afeto Negativo ${ }^{\mathrm{a}}$ & $-0,2 *$ & 0,11 & - & $0,22 *$ & $0,41 * * *$ & 0,03 & $-0,18$ \\
\hline 4. Número ${ }^{\mathrm{a}}$ & $-0,94$ & 0,09 & $0,22 *$ & - & 0,03 & $0,42 * * *$ & 0,06 \\
\hline 5. Impacto ${ }^{\mathrm{a}}$ & $-0,85$ & 0,13 & $0,41 * * *$ & 0,03 & - & $-0,01$ & $-0,2$ \\
\hline 6. Contatos ${ }^{\mathrm{b}}$ & 0,2 & $0,29 * *$ & 0,03 & $0,42 * * *$ & $-0,01$ & - & $-0,12$ \\
\hline 7. Fator de Proximidade ${ }^{b}$ & 0,13 & $-0,05$ & $-0,18$ & 0,06 & $-0,2$ & $-0,12$ & - \\
\hline
\end{tabular}

a Utilizou-se Pearson para correlações nas quais as duas variáveis apresentavam distribuição normal (por exemplo, afeto positivo, afeto negativo, número e impacto de eventos estressores); b Correlações de Spearman foram utilizadas quando pelo menos uma das variáveis apresentou distribuição não normal (por exemplo, satisfação de vida, contatos e fator de proximidade).

$* p<0,05, * * p<0,01$

enquanto que médias mais baixas são encontradas nos afetos negativos. Os afetos positivos e negativos não apresentaram correlação significativa.

\section{Variáveis Independentemente Associadas ao Bem-Estar Subjetivo: A Influência da Idade, Sexo, Eventos Estressores e Rede de Apoio}

Uma análise de regressão linear (método enter) foi realizada a fim de se identificar variáveis independentes (idade, sexo, eventos estressores e rede de apoio) associadas ao BES. Conforme mostra a Tabela 3, no primeiro modelo, verificou-se associação negativa entre idade e satisfação de vida, indicando que os jovens de maior idade têm maior probabilidade de possuir uma satisfação de vida mais baixa. O modelo foi significativo $[\mathrm{F}(2)=12,59, p<0,001]$ e a variância explicada foi de $26 \%$. O modelo não foi significativo quando incluídas as variáveis de eventos estressores e rede de apoio. Verificou-se que os afetos negativos associaram-se ao impacto dos eventos estressores e ao fator de proximidade da rede de apoio, com variância explicada de $21 \%$. O modelo foi significativo $[\mathrm{F}(4)=4,63, p<0,01]$. Isto indicou que quanto maior o impacto do evento estressor e menor a proximidade das relações afetivas, maior a probabilidade de se experienciar afetos negativos. Os afetos positivos não estiveram associados às demais variáveis do presente estudo (em qualquer dos modelos pesquisados).

\section{TABELA 3}

Análise de Regressão Linear para as Variáveis Idade, Sexo, Eventos Estressores (Número e Impacto) e Rede de Apoio (Contatos e Fator de Proximidade) Independentemente Associadas com a Satisfação de Vida, Afeto Positivo e Afeto Negativo

\begin{tabular}{|c|c|c|c|c|c|c|c|c|c|}
\hline & \multicolumn{3}{|c|}{ Satisfação de Vida } & \multicolumn{3}{|c|}{ Afeto Positivo } & \multicolumn{3}{|c|}{ Afeto Negativo } \\
\hline & $B$ & $S E B$ & $B$ & $B$ & $S E B$ & $B$ & $B$ & $S E B$ & $B$ \\
\hline \multicolumn{10}{|l|}{ Modelo 1} \\
\hline Idade & $-0,57$ & 0,23 & $-0,48 * * *$ & $-0,1$ & 0,12 & $-0,1$ & 0,06 & 0,13 & 0,05 \\
\hline \multirow[t]{2}{*}{$\mathrm{Sexo}^{\mathrm{a}}$} & 0,57 & 0,34 & 0,17 & $-0,01$ & 0,37 & $-0,00$ & $-0,84$ & 0,35 & $-0,28$ \\
\hline & \multicolumn{3}{|c|}{$\mathrm{R}^{2}=0,26 ; F=12,59 * * *$} & \multicolumn{2}{|c|}{$\mathrm{R}^{2}=0,01 ; F=0,34$} & & \multicolumn{2}{|c|}{$\mathrm{R}^{2}=0,08 ; F=2,88$} & \\
\hline \multicolumn{10}{|l|}{ Modelo 2} \\
\hline Idade & $-0,53$ & 0,12 & $-0,44 * * *$ & $-0,07$ & 0,12 & $-0,07$ & 0,01 & 0,12 & 0,01 \\
\hline $\operatorname{Sexo}^{\mathrm{a}}$ & 0,18 & 0,37 & 0,05 & 0,13 & 0,39 & 0,04 & $-0,24$ & 0,35 & $-0,08$ \\
\hline Número & $-0,03$ & 0,02 & $-0,12$ & 0,01 & 0,03 & 0,07 & 0,03 & 0,02 & 0,15 \\
\hline Impacto & $-0,24$ & 0,14 & $-0,19$ & 0,21 & 0,14 & 0,2 & 0,47 & 0,13 & $0,39 * * *$ \\
\hline Contatos & 0,01 & 0,00 & 0,15 & 0,01 & 0,00 & 0,21 & $-0,00$ & 0,00 & $-0,08$ \\
\hline \multirow[t]{2}{*}{ Fator de Proximidade } & 0,05 & 0,06 & 0,08 & $-0,02$ & 0,06 & $-0,05$ & $-0,12$ & 0,06 & $-0,22 *$ \\
\hline & \multicolumn{3}{|c|}{$\mathrm{R}^{2}=0,32 ; F=1,45$} & \multicolumn{3}{|c|}{$\mathrm{R}^{2}=0,1 ; F=1,75$} & \multicolumn{3}{|c|}{$\mathrm{R}^{2}=0,21 ; F=4,63 * *$} \\
\hline
\end{tabular}




\section{Discussão}

Os resultados aqui apresentados confirmam resultados de estudos anteriores, que mostram um predomínio de meninos em situação de rua (Alves, 2011; Kulik, Gaetz, Crowe, \& Ford-Jones, 2011; Morais, Neiva-Silva, \& Koller, 2010). Entende-se que esse aspecto relaciona-se aos padrões culturais de gênero, em que é incentivado aos meninos circular pelas ruas, utilizando-as para trabalho e/ou lazer. Já a aparente "invisibilidade" das meninas deve-se ao estímulo à realização de tarefas domésticas, ao cuidado de crianças mais novas e ao maior envolvimento com a exploração sexual (Gontijo \& Medeiros, 2009; Penna, Carinhanha, \& Rodrigues, 2010).

Identificou-se um maior número de jovens em instituições (acolhimentos e serviços abertos) do que propriamente morando nas ruas, fato que sugere a existência de um "novo" perfil de adolescentes em situação de rua. De acordo com esse novo perfil, um menor número deles estariam expostos nas regiões centrais das cidades, seja em virtude da existência de (mais e melhores) programas sociais no interior das comunidades (Finkler, 2011), seja porque estariam envolvidos com o tráfico de drogas nas comunidades, por isso ali ficariam "escondidos". Essa última hipótese foi confirmada neste estudo, quando se pôde verificar, nos relatos de diários de campo, trechos de fala dos jovens que se diziam "perseguidos pelo tráfico", por isto estavam nos centros das cidades ou regiões de beira-mar, como em Salvador e Fortaleza, por exemplo. Por fim, é válido afirmar que a diversidade de locais de coleta de dados corroboram estudos anteriores que identificam diferentes perfis e trajetórias de vinculação com a rua, mostrando a heterogeneidade dessa população (Santana, Doninelli, \& Koller, 2010; Sober et al., 2014).

$\mathrm{Na}$ caracterização dos eventos estressores, os resultados confirmam pesquisas anteriores (Abaid, Dell'Aglio, \& Koller, 2010; Morais, Koller, \& Raffaelli, 2010; Poletto, Koller, \& Dell'Aglio, 2009) que mostram jovens em vulnerabilidade que experienciam com frequência os eventos estressores. Verificou-se ainda que os eventos mais frequentes retratam a transitoriedade e dinâmica de seus percursos. Entende-se que essa característica deve-se a estratégias de sobrevivência, posto que as crianças/adolescentes em situação de rua tendem a transitar por diferentes espaços em busca de melhores condições de vida (Gontijo et al., 2009; Koller, 2011).

Os eventos com médias mais altas de impacto evidenciam a violação de direitos vivenciada pelos jovens e suas famílias. Nota-se que a situação de rua ocorre como um continuum de vulnerabilidade social e não como o único evento estressor de suas vidas (Morais et al., 2012). Dessa forma, a situação de rua tende a ser precedida de inúmeras outras situações negativas (e.g negligência, violência, precariedade material, etc.), as quais os adolescentes escolhem romper através de uma atitude explícita de busca de saúde e bem-estar ao saírem para as ruas.

As análises estatísticas entre eventos estressores e sexo corroboram estudos anteriores (Polleto et al., 2009; Polleto, 2011) que verificaram as meninas com médias mais altas de impacto do que os meninos. Teoricamente, tal fato pode ser explicado pela perspectiva de que elas possuem maior facilidade para se comunicar e reconhecer seus sentimentos e suas manifestações e mais casos de negligência, violência doméstica e abuso sexual serem notificados e/ou verificados em meninas (Poletto, 2011).

As médias de afetos negativos deste estudo referemse de "um pouco" a "mais ou menos". Esta mesma média foi verificada em outro estudo com jovens em situação de rua (Morais et al., 2010). Verifica-se que quando comparado a outros grupos sem vivência de rua, jovens em vulnerabilidade mostram níveis mais baixos de bem-estar (Silva, 2011; Schütz et al., 2014). A correlação positiva dos afetos negativos com número e impacto dos eventos estressores também foi encontrada em Morais et al., (2010) e Poletto (2011). Os jovens deste estudo vivenciaram violência, pobreza entre outros eventos que podem explicar os níveis mais altos dos afetos negativos. Corrobora-se a perspectiva de que a intensidade como são sentidos e percebidos os eventos estressores, aumentam os níveis dos afetos negativos (Paludo, 2008).

Enfatiza-se que os participantes apresentam médias mais altas de afetos positivos do que negativos, corroborando a literatura (Morais et al., 2010; Morais et al., 2012; Paludo, 2008). Diferente de preconceitos e estereótipos que permeiam o imaginário social, os jovens em situação de rua descrevem-se a si mesmos como contentes, espertos, fortes, etc. Tais características indicam que a saída para a rua pode ilustrar a capacidade que os jovens possuem de autogoverno e enfrentamento diante das adversidades (Morais et al., 2010). Segundo Paludo (2008), as emoções positivas não só ajudam as pessoas a sentirem-se bem e felizes, como também contribuem para superar e encontrar significados positivos nas situações estressoras. Neste estudo, os níveis dos afetos positivos indicam que não houve prejuízo na expressão de emoções positivas, mesmo diante dos eventos estressores vividos.

Os resultados relacionados à satisfação de vida apresentaram jovens em situação de rua que avaliaram 
positivamente suas vidas. Constataram-se altas médias de satisfação de vida com correlação significativa entre idade e sexo. Os meninos atribuíram maiores médias à sua satisfação de vida; e essas médias tenderam a ser menores, para ambos os sexos, ao longo das faixas etárias, tal como verificado em pesquisas com jovens institucionalizados (Siqueira et al., 2010; Poletto et al., 2011) e com população jovem em geral (Casas et al., 2013; Sarriera et al., 2012).

Dostaler e Nelson (2003) investigaram a satisfação de vida de ingressantes em instituições para meninas em situação de rua. Os resultados follow-up (3 meses) mostraram que a institucionalização e o atendimento socioassistencial contribuíram para um aumento na satisfação de vida das jovens. A influência da rede de apoio para melhores níveis de BES são apresentados em estudos com jovens (Dell'Aglio \& Siqueira, 2010; McAuley, McKeown, \& Merriman, 2012). Na especificidade da população jovem em vulnerabilidade, assim como os familiares e amigos, os profissionais das instituições evidenciam-se como significativos contatos que influenciam a percepção do bem-estar (Lima \& Morais, 2016).

Neste estudo, verificou-se que número dos eventos estressores e afetos positivos correlacionaram-se positivamente ao número de contatos da rede de apoio. Isto é, quanto mais contatos, os eventos estressores e os afetos positivos foram vivenciados com maior frequência. A análise da rede de apoio incluiu tanto contatos satisfatórios quanto contatos insatisfatórios. Estes podem ter influenciado para a relação do maior número de contatos com o aumento de eventos estressores, visto que são compostos por pessoas que os participantes não possuem proximidade afetiva.

A correlação positiva entre afeto positivo e número de contatos da rede de apoio sugere que a interação social é valorizada pelos jovens e, quando positiva, pode contribuir para vivências agradáveis de bem-estar. Embora se evidencie a necessidade de contextualização da rede de apoio, de modo que cada pessoa, com suas estratégias de enfrentamento, dinâmica familiar, institucional, entre outras características pessoais e relacionais, revelam o processo de influência acerca de experiências positivas e/ou negativas (Morais et al., 2012).

Nas análises entre cidades, verificou-se que Fortaleza teve maior frequência de eventos estressores quando comparada a Salvador. Não houve diferença significativa entre cidades para os componentes do BES, indicando que mesmo Fortaleza com alta ocorrência de eventos estressores não se diferenciou quanto aos níveis de BES. Isto pode sugerir que os participantes de Fortaleza apresentam os piores indicadores de vivência de violência entre outros eventos estressores. No entanto, esses eventos não influenciaram negativamente os afetos positivos e satisfação de vida.

Essa discussão corrobora a literatura que apresenta jovens em vulnerabilidade descrevendo suas vidas de forma positiva (Morais et al., 2012; Poletto et al., 2011). A visão positiva dos jovens apesar das adversidades vividas, além de experiências satisfatórias no cotidiano, pode relacionar-se a uma avaliação da vida atual em comparação a situações anteriores de intensa vulnerabilidade (Raffaelli, Morais, \& Koller, 2013). As estratégias de enfrentamento e a rede de apoio também se configuram como possibilidades de reconhecimento de experiências de bem-estar que favorecem a expressão de emoções e avaliações positivas das suas vidas (Dell'Aglio et al., 2010; Lima et al., 2016).

As análises deste estudo indicaram correlações significativas da satisfação de vida com afetos positivos e negativos, indicando a função reguladora da satisfação de vida sobre as emoções, atuando de forma a aumentar ou diminuir cada uma delas, de acordo com a dimensão cognitiva das pessoas (Giacomoni, 2002). Ademais, a inexistência de correlação entre o afeto positivo e negativo confirma a literatura, quando esta os descreve como componentes independentes (Zanon, et al., 2013).

Por fim, a regressão linear (ver Tabela 3 ) evidenciou que à medida que os jovens completam mais idade, a satisfação de vida tende a diminuir, assim como uma avaliação mais alta do impacto do evento estressor e baixa qualidade das relações afetivas podem aumentar os afetos negativos. Conforme Koller e De Antoni (2004), a relação dos indivíduos com os eventos estressores perpassam diferentes graus de ocorrência, intensidade, frequência, duração e severidade. Assim, não se considera um evento estressor de forma isolada, mas verifica-se que o evento faz parte de um contexto mais complexo e que pode, inclusive, desencadear outros eventos estressores (Morais et al., 2010). Nesse sentido, não apenas o efeito cumulativo dos eventos pode ser prejudicial, mas a percepção do impacto desses eventos é crucial na avaliação de suas implicações ao desenvolvimento e, em específico, a experiência de afetos negativos.

\section{Considerações Finais}

O estudo buscou caracterizar o BES de crianças e adolescentes em situação de rua de três capitais brasileiras: Fortaleza, Porto Alegre e Salvador, verificando os fatores a ele associados (idade, sexo, eventos estressores e rede de apoio). Em geral, 
os resultados indicaram altos níveis de BES entre os participantes, fato que não deve minimizar a importância de intervenções direcionadas a esse público, tão violado em seus direitos básicos.

Conforme mostraram as análises dos eventos estressores, jovens em situação de rua possuem uma trajetória marcada por múltiplas adversidades. Políticas públicas que atuem no enfrentamento de riscos ao desenvolvimento dos jovens fazem-se necessárias e urgentes, tanto em caráter emergencial quanto de prevenção e promoção de saúde. Para tanto, a valorização dos recursos pessoais e relacionais acerca dos contextos nos quais os jovens em situação de rua estão inseridos e das pessoas com quem convivem precisam ganhar lugar de destaque.

A elucidação dos seus aspectos positivos pode ser uma forma de contribuir para um trabalho que vise à superação das adversidades. Fala-se, assim, de uma mudança de olhar, no qual ao invés de intervenções centradas na vulnerabilidade e no risco em si, acentuese uma atuação fortalecedora de aspectos positivos, como o BES. Visto que, de acordo com os resultados desse estudo, os jovens apesar de vivenciarem eventos estressores, não se limitaram a vivências, sentimentos e emoções negativas. Cabe, portanto, a descoberta e ênfase das forças propulsoras de desenvolvimento que facilitam a expressão de recursos positivos e, concomitante, enfrentamento e superação dos riscos.

Quanto às limitações e sugestões para pesquisas futuras, este estudo de delineamento transversal possibilitou o conhecimento das variáveis investigadas num momento específico do tempo. Uma pesquisa de natureza longitudinal poderia compreender os processos positivos dos jovens ao longo do tempo, verificando possíveis implicações dos eventos estressores em relação ao BES em momentos posteriores.

Finalmente, espera-se ter contribuído para uma visão mais complexa e positiva do desenvolvimento em contextos adversos, uma vez que se tentou conhecer indicadores positivos que potencializam o desenvolvimento de jovens em situação de rua. Em se tratando de uma população tradicionalmente descrita a partir do levantamento de múltiplos indicadores de risco, considera-se que esse esforço inicial representa um passo importante no (longo e interessante) caminho que ainda precisa ser percorrido nesse campo de estudos sobre desenvolvimento "positivo" em contextos de vulnerabilidade social.

\section{Referências}

Abaid, W., Lieberknecht, J., Dell'aglio, D. D., \& Koller, S. H. (2010). Preditores de sintomas depressivos em crianças e adolescentes institucionalizados. Universitas Psychologica, 9(1), 199-212. http://dx.doi.org/10.11144/237

Alves, P. B. (2011). O estudo sobre crianças em situação de rua na perspectiva da teoria dos sistemas ecológicos: contribuições teóricas e metodológicas. In S. H. Koller (Ed.). Ecologia do desenvolvimento humano: Pesquisa e intervenção no Brasil (pp. 125-145). São Paulo: Casa do Psicólogo.

Brito, R. \& Koller, S. H. (1999). Desenvolvimento humano e redes de apoio social e afetivo. In A. M. Carvalho (Ed.). O mundo social da criança: Natureza e cultura em ação (pp. 115-129). São Paulo: Casa do Psicólogo.

Casas, F., Fernández-Artamendi, S., Montserrat, C., Bravo, A., Bertrán, I., \& Dell Valle, J. F. (2013). El bienestar subjetivo en la adolescencia: Estudio comparativo de dos Comunidades Autónomas en Espa-a. Anales de Psicología, 29(1), 148-158. http://dx.doi.org/10.6018/analesps.29.1.145471

Cecconello, A. M. \& Koller, S. H. (2003). Inserção ecológica na comunidade: Uma proposta metodológica para o estudo de famílias em situação de risco. Psicologia: Reflexão e Crítica, 16(3), 515-524. http://dx.doi.org/10.1590/S010279722003000300010

Cervantes, S. C. \& Sosa, J. J. (2015). Ni-as y adolescentes en riesgo de calle: Bienestar subjetivo y salud mental. Revista CES Psicología, 8(1), 120-133.

Dell'Aglio, D. D., \& Siqueira, A. C. (2010). Preditores de satisfação de vida de jovens em situação de vulnerabilidade no sul do Brasil. Psicodebate, 10, 213-230.

Diener, E., Emmons, R. A., Larsen, R. J., \& Griffin, S. (1985). The satisfaction with life scale. Journal of Personality Assessment, 49(1), 71-75. http://dx.doi.org/10.1207/s15327752jpa4901_13

Diener, E. (2012). New findings and future directions for subjective well-being research. The American Psychologist, 67(8), 590-597. http://dx.doi.org/10.1037/a0029541

Dostalter, T. \& Nelson, G. (2003). A process and outcome evaluation of a shelter for homeless young women. Canadian Journal of Community Mental Health, 22(1), 99-112. http://dx.doi.org/10.7870/cjcmh-2003-0007

Finkler, L. (2011). Avaliação de um projeto social para crianças e adolescentes em situação de rua e suas família [Tese de Doutorado não publicada], Universidade Federal do Rio Grande do Sul, Porto Alegre, RS.

Giacomoni, C. (2002). Bem-estar subjetivo infantil: conceito de felicidade e construção de instrumentos para avaliação [Tese de Doutorado não publicada], Universidade Federal do Rio Grande do Sul, Porto Alegre, RS. 
Gontijoa, D. T. \& Medeiros, M. (2009). Adolescência, gênero e processo de vulnerabilidade/desfiliação social: Compreendendo as relações de gênero para adolescentes em situação. Revista Baiana de Saúde Pública, 33(4), 605-617.

Hoppe, M. (1998). Redes de apoio social e afetivo de crianças em situação de risco [Dissertação de Mestrado não publicada], Universidade Federal do Rio Grande do Sul, Porto Alegre, RS.

Kristensen, C. H., Leon, J. S., D’Incao, D. B., \& Dell'Aglio, D. D. (2004). Análise da frequência e do impacto de eventos estressores em uma amostra de adolescentes. Interação em Psicologia, 8(1), 45-55.

Koller, S. H. \& Hutz, C. (1996). Meninos e meninas em situação de rua: Dinâmica, diversidade e definição. Coletâneas da ANPEPP, 1, 11-34.

Koller, S. H. \& De Antoni, C. (2004). Violência familiar: Uma visão ecológica. In S. H. Koller (Ed.). Ecologia do desenvolvimento humano: Pesquisa e intervenção no Brasil (pp. 293-310). São Paulo: Casa do Psicólogo.

Koller, S. H. (2011). Psicólogos de rua. In S. H. Koller (Ed.). Ecologia do desenvolvimento humano: Pesquisa e intervenção no Brasil (pp. 23-46). São Paulo: Casa do Psicólogo.

Kulik, D. M., Gaetz, S., Crowe, C., \& Ford-Jones, E. L. (2011). Homeless youth's overwhelming health burden: A review of the literature. Pediatric Child Health, 16(6), e43-e47.

Lima, R. F. F., \& Morais, N. A. (2016). Caracterização qualitativa do bem-estar subjetivo de crianças e adolescentes em situação de rua. Temas em Psicologia, 24(1), 1-15. http://dx.doi.org/10.9788/TP2016.1-01

Masten, A. S. \& Garmezy, N. (1985). Risk, vulnerability and protective factors in developmental psycho-pathology. In B. B. Lahey \& A. E. Kazdin (Eds.). Advances in clinical child psychology (pp.1-52). New York: Plenum Press. http:// dx.doi.org/10.1007/978-1-4613-9820-2_1

McAuley, C., McKeown, C., \& Merriman, B. (2012). Spending time with family and friends: Children's views on relationships and shared activities. Child Indicators Research, 5(3), 449-467. http://dx.doi.org/10.1007/s12187-0129158-2

Morais, N. A., Koller, S. K, \& Raffaelli, M. (2010). Eventos estressores e indicadores de ajustamento entre adolescentes em situação de vulnerabilidade social no Brasil. Universitas Psychologica, 9(3), 315-330. http://dx.doi.org/10.11144/474

Morais, N. A., Neiva-Silva, L., \& Koller, S. H. (2010). Crianças e adolescentes em situação de rua: História, caracterização e modo de vida. In N. A. Morais, L. Neiva-Silva, \& S. H. Koller (Eds.). Endereço desconhecido: Crianças e adolescentes em situação de rua (pp. 35-61). São Paulo: Casa do Psicólogo.

Morais, N. A., Koller, S. H., \& Raffaelli, M. (2012). Rede de apoio, eventos estressores e mau ajustamento na vida de crianças e adolescentes em situação de vulnerabilidade social. Universitas Psychologica, 11(3), 779-791. http://dx.doi. org/10.11144/779

Morais, N. A., Raffaelli, M., \& Koller, S. H. (2012). Adolescentes em situação de vulnerabilidade social e o continuum risco-proteção. Avances em Psicologia Latinoamericana, 30(1), 122-140.

Paludo, S. (2008). Emoções morais e gratidão: Uma nova perspectiva sobre o desenvolvimento de jovens que vivem em situação de risco pessoal e social [Tese de Doutorado não publicada], Universidade Federal do Rio Grande do Sul, Porto Alegre, RS.

Penna, L. H., Carinhanha, J. I., \& Rodrigues, R. F. (2010). Violência vivenciada pelas adolescentes em situação de rua na ótica dos profissionais cuidadores do abrigo. Revista Eletrônica de Enfermagem, 12(2), 301-307.

Polletto, M., Koller, S. H., \& Dell'Aglio, D. D. (2009). Eventos estressores em crianças e adolescentes em situação de vulnerabilidade social de Porto Alegre. Ciência e Saúde Coletiva, 14(2), 455-466. http://dx.doi.org/10.1590/S141381232009000200014

Poletto, M. (2011). Bem-estar subjetivo: um estudo longitudinal com crianças e adolescentes em situação de vulnerabilidade social [Tese de Doutorado não publicada], Universidade Federal do Rio Grande do Sul, Porto Alegre, RS.

Poletto, M. \& Koller, S. H. (2011). Subjective well-being in socially vulnerable children and adolescents. Psicologia: Reflexão e Crítica, 24(3), 476-484. http://dx.doi.org/10.1590/S0102-79722011000300008

Prati, L. E., Couto, M. C., Moura, A., Poletto, M., \& Koller, S. H. (2008). Revisando a inserção ecológica: Uma proposta de sistematização. Psicologia: Reflexão e Crítica, 21(1), 160-169. http://dx.doi.org/10.1590/S0102-79722008000100020

Raffaelli, M., Koller, S. H., \& Morais, N. A. (2007). Assessing the development of Brazilian street youth. Vulnerable Children and Youth Studies, 2, 154-164. http://dx.doi.org/10.1080/17450120701403128

Raffaelli, M., Morais, N. A., \& Koller, S. H. (2013). Children at risk: The case of Latin American street youth. In: A. Ben-Arieh, F. Casas, I. Frones, \& J. E. Korbin (Eds.). Handbook of child well-being: Theories, methods and policies in global perspective (pp. 2653-2668). New York: Springer Dordrecht Heidelberg. http://dx.doi.org/10.1007/978-90481-9063-8

Rees, G., Goswami, H., \& Bradshaw, J. (2010). Developing an index of children's subjective well-being. London: The Children's Society.

Samuelsson, M., Thernlund, G., \& Ringström, J. (1996). Using the five field map to describe the social network of children: A methodological study. International Journal of Behavioral Development, 19, 327-346. http://dx.doi. org/10.1080/016502596385811 
Santana, J. P., Doninelli, T. M., \& Koller, S. H. (2010). Trajetória de vinculação institucional de crianças e adolescentes em situação de rua. In A. Morais, L. Neiva-Silva, \& S. H. Koller (Eds.). Endereço desconhecido: crianças e adolescentes em situação de rua (pp. 405-419). São Paulo, Casa do Psicólogo.

Sarriera, J. C., Saforcada, E., Tonon, G., La Vega, L. R., Mozobancyk, S., Bedin, L. M. (2012). Bienestar subjetivo de los adolescentes: Un estudio comparativo entre Argentina y Brasil. Psychosocial Intervention, 21(3), 273-280. http:// dx.doi.org/10.5093/in2012a24

Schütz, F., Sarriera, J., Bedin, L. \& Montserrat, C. (2015). Subjective well-being of children in residential care: Comparison between children in institutional care and children living with their families. Psicoperspectivas, 14(1), 19-30. http://dx.doi.org/10.5027/psicoperspectivas-vol14-issue1-fulltext-517

Segabinazi, J. D., Giacomoni, C. H., Dias, A. C., Teixeira, M. A., \& Moraes, D. A. (2010). Desenvolvimento e validação preliminar de uma escala multidimensional de satisfação de vida para adolescentes. Psicologia: Teoria e Pesquisa, 26(4), 653-659. http://dx.doi.org/10.1590/S0102-37722010000400009

Silva, M. J. (2011). O bem-estar subjectivo de adolescentes institucionalizados [Tese de Doutorado não publicada], Universidade de Lisboa, Lisboa, Portugal.

Siqueira, A. C., Tubino, C. de L., Schwarz, C., \& Dell’Aglio, D. D. (2009). Percepção das figuras parentais na rede de apoio de crianças e adolescentes institucionalizados. Arquivos Brasileiros de Psicologia, 61(1), 176-190.

Siqueira, A. C., \& Dell'Aglio, D. D. (2010). Crianças e adolescentes institucionalizados: Desempenho escolar, satisfação de vida e rede de apoio social. Psicologia: Teoria e Pesquisa, 26(3), 407-415. http://dx.doi.org/10.1590/S010237722010000300003

Sorber, R., Winston, S., Koech, J., Ayuku, D., Hu, L., Hogan, J., \& Braitstein, P. (2014) Social and economic characteristics of street youth by gender and level of street involvement in Eldoret, Kenya. PLoS ONE, 9(5): e97587. http://dx.doi. org/10.1371/journal.pone.0097587

Zanon, C., Bastianello, M. R., Pacico, J. C., \& Hutz, C. S. (2013). Desenvolvimento e validação de uma escala de afetos positivos e negativos. Psico-USF, 18(2), 193-201. http://dx.doi.org/10.1590/S1413-82712013000200003

Apoio financeiro:

Trabalho realizado com apoio da Jacobs Foundation e Edital Universal do Conselho Nacional de Desenvolvimento Científico e Tecnológico (CNPq, 2011).

Autores:

Rebeca Fernandes Ferreira Lima - Doutoranda, Universidade de Fortaleza.

Normanda Araujo de Morais - Doutora, Universidade de Fortaleza.

Endereço para correspondência:

Rebeca Fernandes Ferreira Lima

<rebecafflima@gmail.com>

Recebido em: 27.02.2015

Aceito em: 11.11.2015 